Pacific Journal of Mathematic 


\title{
TWO RELATED INTEGRALS OVER SPACES OF CONTINUOUS FUNCTIONS
}

\author{
R. H. Cameron and D. A. Storvick
}

\begin{abstract}
In this paper the authors evaluate Yeh-Wiener integrals (which apply to functionals of a variable continuous function of two arguments) in terms of multiple Wiener integrals (which apply to functionals of several variable continuous functions of one argument). First somewhat specialized cases are given where the multiplicity of the Wiener integral is finite, and then quite general Yeh-Wiener integrals are evaluated in terms of limits of $n$-fold Wiener integrals as $n \rightarrow \infty$.
\end{abstract}

Introduction. James Yeh [5] ${ }^{1}$ defined Wiener measure in the space $C_{2}[S]$ of continuous real valued functions of two variables defined on the square $S: 0 \leqq s \leqq 1,0 \leqq t \leqq 1$ and vanishing whenever $s$ or $t$ equals zero. More recently James Kuelbs [3,4] extended Yeh's integral to integration over $C_{2}[X]$, the space of continuous real valued functions on any compact subset $X$ of the plane. Kuelbs also defined a similar integral over spaces of functions of several variables and even infinitely many variables [4].

In the present paper we shall consider integration over $C_{2}[X]$ in the case where $X$ is the rectangle $R=\{(s, t) \mid a \leqq s \leqq b, \alpha \leqq t \leqq \beta\}$. We note that this is closely connected with Yeh's integral over $C_{2}[S]$ and that Kuelbs has given a formula for relating integrals over $C_{2}[R]$ with integrals over $C_{2}[S],[3, \mathrm{p} .18]$.

Yeh's measure as applied to the space

$$
\begin{gathered}
C_{2}[R] \equiv\{x(\cdot, \cdot) \mid x(a, t)=x(s, \alpha)=0, x(s, t) \\
\text { continuous for } a \leqq s \leqq b, \alpha \leqq t \leqq \beta\}
\end{gathered}
$$

is defined as follows. Let $a=s_{0}<s_{1}<\cdots<s_{m}=b$, and $\alpha=t_{0}<$ $t_{1}<\cdots<t_{n}=\beta$ be subdivisions of $[a, b]$ and $[\alpha, \beta]$ respectively and let $-\infty \leqq P_{j, k} \leqq Q_{j, k} \leqq+\infty$ be given for $j=1, \cdots, m$ and $k=$ $1, \cdots, n$. Then

$$
I=\left\{x \in C_{2}[R] \mid P_{j, k}<x\left(s_{j}, t_{k}\right) \leqq Q_{j, k} \text { for } j=1, \cdots, m, k=1, \cdots, n\right\}
$$

will be called an "interval" in $C_{2}[R]$. He defines the measure of the interval $I$ by

\footnotetext{
${ }^{1}$ See also reference to Kitagawa in [5].
} 


$$
\begin{gathered}
m(I)=\pi^{-m n / 2}\left[\left(s_{1}-s_{0}\right) \cdots\left(s_{m}-s_{m-1}\right)\right]^{-n / 2}\left[\left(t_{1}-t_{0}\right) \cdots\left(t_{n}-t_{n-1}\right)\right]^{-m / 2} \\
\cdot \int_{P_{m, n}}^{Q_{m, n}} \cdots \int_{P_{1,1}}^{(m n)} \exp \left\{-\sum_{j=1}^{m} \sum_{k=1}^{n} \frac{\left[u_{j, k}-u_{j-1, k}-u_{j, k-1}+u_{j-1, k-1}\right]^{2}}{\left(s_{j}-s_{j-1}\right)\left(t_{k}-t_{k-1}\right)}\right\} d u_{1,1} \cdots d u_{m, n}
\end{gathered}
$$

where $u_{0, k} \equiv u_{j, 0} \equiv 0$ for $j=1, \cdots, m ; k=1, \cdots, n$.

This measure is countably additive on the set of intervals in $C_{2}[R]$ and can be extended in the usual way to the sigma-algebra of sets generated by the intervals and can then be further extended so as to be a complete measure. Thus "Yeh-Wiener measurable set" and its "measure" are defined in $C_{2}[R]$.

The integrals of functionals integrable with respect to this measure will be called "Yeh-Wiener integrals".

In Theorem 1 of the present paper we establish a formula for evaluating in terms of a Wiener integral the Yeh-Wiener integral of a functional of $x(\cdot, \cdot)$ which actually depends solely on the values of $x$ on one horizontal line.

Theorem 2 treats the case of a functional depending only on the values of $x$ on a finite number of horizontal lines.

Theorem 4 deals with the case of a functional depending only on the values of $x$ on the two (perpendicular) free edges of $R$. Examples are given to show how Theorem 4 can be used to evaluate Yeh-Wiener integrals of specific functionals.

Finally in Theorem 5 we consider a class of functionals that may depend on the values which $x$ assumes at all points of the rectangle $R$ and not only on the values $x$ assumes on some restricted set.

1. The one line theorem. Let $C_{1}[a, b] \equiv\{y(\cdot) \mid y(a)=0, y(t)$ continuous on $[a, b]\}$, let $R \equiv[a, b] \times[\alpha, \beta]$ and let

$$
\begin{gathered}
C_{2}[R] \equiv\{x(\cdot, \cdot) \mid x(\alpha, t)=x(s, \alpha)=0, x(s, t) \\
\text { continuous for } a \leqq s \leqq b, \alpha \leqq t \leqq \beta\} .
\end{gathered}
$$

THeOREm 1. Let $\alpha<\gamma \leqq \beta$, and let $f(\cdot)$ be a real or complex valued functional defined on $C_{1}[a, b]$ such that $f(\sqrt{(\gamma-\alpha) / 2} y)$ is a Wiener measurable functional of $y$ on $C_{1}[a, b]$. Then $f(x(\cdot, \gamma))$ is a Yeh-Wiener measurable functional of $x(\cdot, \cdot)$ on $C_{2}[R]$ and

$$
\int_{C_{2}[R]} f(x(\cdot, \gamma)) d x=\int_{C_{1}[a, b]} f\left(\sqrt{\frac{\gamma-\alpha}{2}} y\right) d y,
$$

where the existence of either integral implies the existence of the other and their equality.

Proof. Let $g(y) \equiv f(\sqrt{(\gamma-\alpha) / 2} y)$. Then it suffices to prove that $g(\sqrt{2 /(\gamma-\alpha)} x(\cdot, \gamma))$ is Yeh-Wiener measurable and that 


$$
\int_{C_{2}[R]} g\left(\sqrt{\frac{2}{\gamma-\alpha}} x(\cdot, \gamma)\right) d x=\int_{C_{1}[a, b]} g(y) d y
$$

where the existence of either implies the existence of the other and their equality.

Case I. Let us consider a subdivision $a=s_{0}<s_{1}<\cdots<s_{m}=b$ and let $g(y)=\chi_{I}(y)$ where $I$ is the interval

$$
I=\left\{y \in C_{1}[a, b] \mid-\infty \leqq z_{i}<y\left(s_{i}\right) \leqq w_{i} \leqq+\infty, i=1, \cdots, m\right\}
$$

so that

$$
g\left(\sqrt{\frac{2}{\gamma-\alpha}} x(\cdot, \gamma)\right)=\chi_{I}\left(\sqrt{\frac{2}{\gamma-\alpha}} x(\cdot, \gamma)\right)=\chi_{K}(x(\cdot, \cdot))
$$

where

$$
\begin{aligned}
K & =\left\{x \in C_{2}[R] \mid-\infty \leqq \sqrt{\frac{\gamma-\alpha}{2}} z_{i}<x\left(s_{i}, \gamma\right) \leqq \sqrt{\frac{\gamma-\alpha}{2}} w_{i}\right. \\
& \leqq+\infty, i, \cdots, m\} .
\end{aligned}
$$

Thus in this case, $g(\sqrt{2 /(\gamma-\alpha)} x(\cdot, \gamma))$ is Yeh-Wiener measurable on $C_{2}[R]$ (see Definition (2.1) of [4, p. 434]).

Because $g(\sqrt{2 /(\gamma-\alpha)} x(\cdot, \gamma))$ is the characteristic functional of an interval, the left member of equation (1.2) equals the measure of the interval $K$, i.e.,

$$
\begin{aligned}
& \int_{C_{2}[R]} g\left(\sqrt{\frac{2}{\gamma-\alpha}} x(\cdot, \gamma)\right) d x=\int_{C_{2}[R]} \chi_{K}(x(\cdot, \cdot)) d x \\
= & {\left[\pi^{m}\left(s_{1}-s_{0}\right)\left(s_{2}-s_{1}\right) \cdots\left(s_{m}-s_{m-1}\right)(\gamma-\alpha)^{m}\right]^{-1 / 2} \int_{\sqrt{(\gamma-\alpha) / 2} z_{m}}^{\sqrt{(\gamma-\alpha) / 2} w_{m}}(m) \int_{\sqrt{(\gamma-\alpha) / 2 z_{1}}}^{\sqrt{(\gamma-\alpha) / 2} w_{1}} } \\
& \cdot \exp \left\{-\sum_{i=1}^{m} \frac{\left(u_{i}-u_{i-1}\right)^{2}}{\left(s_{i}-s_{i-1}\right)(\gamma-\alpha)}\right\} d u_{1} \cdots d u_{m},
\end{aligned}
$$

where $u_{0} \equiv 0$.

The right hand member of (1.2) can be evaluated in the following manner,

$$
\begin{aligned}
& \int_{C_{1}[a, b]} g(y) d y=\int_{C_{1}[a, b]} \chi_{I}(y) d y=\left[(2 \pi)^{m}\left(s_{1}-s_{0}\right) \cdots\left(s_{m}-s_{m-1}\right)\right]^{-1 / 2} \int_{z_{m}}^{w_{m}} \cdots \int_{z_{1}}^{(m)} \int_{1}^{w_{1}} \\
& \cdot \exp \left\{-\sum_{i=1}^{m} \frac{\left(v_{i}-v_{i-1}\right)^{2}}{2\left(s_{i}-s_{i-1}\right)}\right\} d v_{1} \cdots d v_{m},
\end{aligned}
$$

where $v_{0} \equiv 0$. If we set $v_{i}=\sqrt{2 /(\gamma-\alpha)} u_{i}$ we obtain (1.2) and hence (1.1).

Case II. Let $g(y)=\chi_{\Omega}(y)$ where $\Omega$ is the union of the disjoint intervals $I_{1}, I_{2}, \cdots$. Then by Case $I$, we have 


$$
\int_{C_{2}[R]} \chi_{I_{k}}\left(\sqrt{\frac{2}{\gamma-\alpha}} x(\cdot, \gamma)\right) d x=\int_{C_{1}[a, b]} \chi_{I_{k}}(y) d y
$$

including the measurability of the left hand integrand. The functional obtained by summing over $k$ is Yeh-Wiener measurable, i.e.,

$$
\sum_{k=1}^{\infty} \chi_{I_{k}}\left(\sqrt{\frac{2}{\gamma-\alpha}} x(\cdot, \gamma)\right) \equiv \chi_{\Omega}\left(\sqrt{\frac{2}{\gamma-\alpha}} x(\cdot, \gamma)\right)
$$

is Yeh-Wiener measurable. Then summing the integrals we have

$$
\int_{C_{2}[R]} \chi_{\Omega}\left(\sqrt{\frac{2}{\gamma-\alpha}} x(\cdot, \gamma)\right) d x=\int_{C_{1}[a, b]} \chi_{\Omega}(y) d y \text {. }
$$

Thus (1.2) holds in this case.

Case III. Let $g(y)=\chi_{\lrcorner}(y)$ where $\Delta$ is a countable intersection of sets $\Omega$ of the type considered in Case II. Since finite intersections of such sets are of the same type, we can set

$$
\Delta=\bigcap_{k=1}^{\infty} \Omega_{k}
$$

where $\Omega_{1} \supset \Omega_{2} \supset \Omega_{3} \supset \cdots$ and each $\Omega_{k}$ is of the type considered in Case II. Thus

$$
g(y)=\lim _{k \rightarrow \infty} \chi_{\Omega_{k}}(y),
$$

and $g(y)$ is Yeh-Wiener measurable. If we now apply (1.2) to $\chi_{\Omega_{k}}$ and take limits we obtain (1.2) for $g(y)=\chi_{4}(y)$, including the measurability of $g(\sqrt{2 /(\gamma-\alpha)} x(\cdot, \gamma))$.

Case IV. Let $g(y)=\chi_{N}(y)$ where $N$ is a Wiener null set. Let $N_{1}$ be a Wiener null set of the type discussed in Case III such that $N_{1} \supset N$. Then (1.2) holds for $\chi_{N_{1}}(y)$ and we have

$$
\int_{C_{2}[R]} \chi_{N_{1}}\left(\sqrt{\frac{2}{\gamma-\alpha}} x(\cdot, \gamma)\right) d x=\int_{C_{1}[a, b]} \chi_{N_{1}}(y) d y=0 \text {, }
$$

including the measurability of the left hand integrand which we now know to be Yeh-Wiener almost everywhere zero. Thus

$$
\chi_{N}\left(\sqrt{\frac{2}{\gamma-\alpha}} x(\cdot, \gamma)\right)
$$

is also Yeh-Wiener almost everywhere zero and (1.2) holds.

Case V. Let $g(y)=\chi_{E}(y)$ where $E$ is any Wiener measurable set. Then $E=\Delta-N$ where $\Delta$ and $N$ are sets of the type considered in 
Cases III and IV. By applying (1.2) to $\Delta$ and to $N$ we obtain (1.2) for $E$ including the measurability of the left hand integrand.

Case VI. Let $g(y)$ be a simple functional (with respect to Wiener measure). Then $g(y)$ is a linear combination with constant coefficients of a finite number of functionals of the type considered in Case V. Hence (1.2) holds.

Case VII. Let $g(y)$ be a real nonnegative Wiener measurable functional. Then $g(y)$ is the limit of a monotone increasing sequence of simple functionals and (1.2) follows from Case VI by monotone convergence.

Case VIII. General case: Because any complex valued functional can be decomposed into its real and imaginary parts and they into their positive and negative parts, the theorem is proved.

2. The $n$-parallel lines theorem. Having obtained a formula for Yeh-Wiener integrals where the functional of $x(\cdot, \cdot)$ actually depends only on the values of $x(\cdot, \gamma)$, i.e., on the values of $x$ on one horizontal line of the fundamental rectangle $R$, it is natural to inquire next concerning functionals that depend solely on the values of $x$ on a finite number of horizontal lines, i.e., functionals of the form

$$
F(x)=f\left[x\left(\cdot, t_{1}\right), x\left(\cdot, t_{2}\right), \cdots, x\left(\cdot, t_{n}\right)\right] .
$$

One might expect to obtain the Yeh-Wiener integral of $F$ as an $n$-fold Wiener integral over the product of $n$ Wiener spaces. Since it is not immediately apparent what the formula should be, we begin with the case where $f$ depends on the values of the $y_{k}(\cdot)$ at a finite number of points. Thus we let

$$
\begin{aligned}
& f\left[y_{1}, \cdots, y_{n}\right] \\
& \quad=\varphi\left[y_{1}\left(s_{1}\right), \cdots, y_{1}\left(s_{m}\right) ; y_{2}\left(s_{1}\right), \cdots, y_{2}\left(s_{m}\right) ; \cdots ; y_{n}\left(s_{1}\right), \cdots, y_{n}\left(s_{m}\right)\right]
\end{aligned}
$$

where

$$
\varphi\left(u_{1,1}, u_{2,1}, \cdots, u_{m, 1} ; \cdots ; u_{1, n}, \cdots, u_{m, n}\right) \equiv \varphi(U)
$$

is defined on $R^{m n}$ and $U$ denotes the rectangular array $\left\{u_{i, j}\right\}_{i=1, \ldots, m ; j=1, \ldots, n}$. Then from (2.0) and (2.1) we have

$$
F(x)=\varphi\left(\left\{x\left(s_{i}, t_{j}\right)\right\}_{\substack{i=1, \ldots, m \\ j=1, \ldots, m}}\right)
$$

Integrating over $C_{2}[R]$ and evaluating the Yeh-Wiener integral we have 


$$
\int_{C_{2}[R]} F(x) d x=\left[\pi^{m n} \prod_{i=1}^{m} \prod_{j=1}^{n}\left(s_{i}-s_{i-1}\right)\left(t_{j}-t_{j-1}\right)\right]^{-1 / 2}
$$

$$
\int_{R^{m n}} \varphi(U) \exp \left\{-\sum_{i=1}^{m} \sum_{j=1}^{n} \frac{\left(u_{i, j}-u_{i-1, j}-u_{i, j-1}+u_{i-1, j-1}\right)^{2}}{\left(s_{i}-s_{i-1}\right)\left(t_{j}-t_{j-1}\right)}\right\} d U
$$

where $d U=d u_{1,1} \cdots d u_{m, n}$, where $u_{0, j} \equiv u_{i, 0} \equiv 0$.

We now make the transformation

$$
v_{i, j}=\sqrt{\frac{2}{t_{j}-t_{j-1}}}\left(u_{i, j}-u_{i, j-1}\right)
$$

so

$$
u_{i, j}=\sqrt{\frac{t_{1}-\alpha}{2}} v_{i, 1}+\sqrt{\frac{t_{2}-t_{1}}{2}} v_{i, 2}+\cdots+\sqrt{\frac{t_{j}-t_{j-1}}{2}} v_{i, j}
$$

and obtain

$$
\begin{aligned}
& \int_{C_{2}[R]} F(x) d x=\left[(2 \pi)^{m} \prod_{i=1}^{m}\left(s_{i}-s_{i-1}\right)\right]^{-n / 2} \\
& \int_{R^{m n}} \varphi\left(\left\{\sqrt{\frac{t_{1}-\alpha}{2}} v_{i, 1}+\sqrt{\frac{t_{2}-t_{1}}{2}} v_{i, 2}+\cdots\right.\right. \\
& \left.\left.+\sqrt{\frac{t_{j}-t_{j-1}}{2}} v_{i, j}\right\}_{\substack{i=1, \ldots, m \\
j=1, \cdots, n}}\right) .
\end{aligned}
$$

$\prod_{j=1}^{n} \exp \left\{-\frac{1}{2} \sum_{i=1}^{m} \frac{\left(v_{i, j}-v_{i-1, j}\right)^{2}}{\left(s_{i}-s_{i-1}\right)}\right\} d V$, where $d V=d v_{1,1} \cdots d v_{m, n}$,

$$
\text { where } v_{0, j} \equiv 0 \text {. }
$$

For each fixed $j$, the sums in the exponential are those which would occur in the evaluation of a Wiener integral, and so we see that the whole expression is the evaluation of an $n$-fold Wiener integral. Thus

$$
\begin{aligned}
& \int_{C_{2}[R]} F(x) d x=\int_{C_{1}[a, b]} \cdots \int_{C_{1}[a, b]} \varphi\left(\left\{\sqrt{\frac{t_{1}-\alpha}{2}} y_{1}\left(s_{i}\right)\right.\right. \\
& \left.\left.+\sqrt{\frac{t_{2}-t_{1}}{2}} y_{2}\left(s_{i}\right)+\cdots+\sqrt{\frac{t_{j}-t_{j-1}}{2}} y_{j}\left(s_{i}\right)\right\}_{\substack{i=1, \cdots, m \\
j=1, \cdots, n}}\right) d y_{1} \cdots d y_{n} .
\end{aligned}
$$

We shall use the following notation for the cartesian product of $n$ Wiener spaces $\stackrel{n}{\times} C_{1}[a, b] \equiv C_{1}[a, b] \times \stackrel{(n)}{\cdots} \times C_{1}[a, b]$.

We have given the motivation for the following theorem:

TheOREM 2. Let $\alpha=t_{0}<t_{1}<\cdots<t_{n}=\beta$ and let $f\left[y_{1}, \cdots, y_{n}\right]$ be a real or complex valued functional defined on $\stackrel{n}{\times} C_{1}[a, b]$ such that 


$$
\begin{aligned}
& f\left[\sqrt{\frac{t_{1}-t_{0}}{2}} y_{1}, \sqrt{\frac{t_{1}-t_{0}}{2}} y_{1}\right. \\
& \left.\quad+\sqrt{\frac{t_{2}-t_{1}}{2}} y_{2}, \cdots, \sqrt{\frac{t_{1}-t_{0}}{2}} y_{1}+\cdots+\sqrt{\frac{t_{n}-t_{n-1}}{2}} y_{n}\right]
\end{aligned}
$$

is a Wiener measurable functional of $\left(y_{1}, \cdots, y_{n}\right)$ on $\stackrel{n}{\times} C_{1}[a, b]$. Then $f\left[x\left(\cdot, t_{1}\right), \cdots, x\left(\cdot, t_{n}\right)\right]$ is a Yeh-Wiener measurable functional of $x(\cdot, \cdot)$ on $C_{2}[R]$ and

$$
\begin{aligned}
& \int_{C_{2}[R]} f\left[x\left(\cdot, t_{1}\right), \cdots, x\left(\cdot, t_{n}\right)\right] d x \\
& =\int_{\times C_{1}[a, b]}^{n} f\left[\sqrt{\frac{t_{1}-t_{0}}{2}} y_{1}, \sqrt{\frac{t_{1}-t_{0}}{2}} y_{1}\right. \\
& +\sqrt{\frac{t_{2}-t_{1}}{2}} y_{2}, \cdots, \sqrt{\frac{t_{1}-t_{0}}{2}} y_{1}+\cdots \\
& \left.+\sqrt{\frac{t_{n}-t_{n-1}}{2}} y_{n}\right] d\left(y_{1} \times \cdots \times y_{n}\right)
\end{aligned}
$$

where the existence of either integral implies the existence of the other and their equality.

Proof. ${ }^{2}$ Let

$$
g\left(y_{1}, \cdots, y_{n}\right)=f\left(\sqrt{\frac{t_{1}-t_{0}}{2}} y_{1}, \cdots, \sum_{k=1}^{n} \sqrt{\frac{t_{k}-t_{k-1}}{2}} y_{k}\right)
$$

Making the substitution $z_{\nu}=\sum_{k=1}^{\nu} \sqrt{\left(t_{k}-t_{k-1}\right) / 2} y_{k}$, we have

$$
f\left(z_{1}, \cdots, z_{n}\right)=g\left(\sqrt{\frac{2}{t_{1}-t_{0}}}\left(z_{1}-z_{0}\right), \cdots, \sqrt{\frac{2}{t_{n}-t_{n-1}}}\left(z_{n}-z_{n-1}\right)\right) .
$$

Thus it suffices to prove that if $g\left(y_{1}, \cdots, y_{n}\right)$ is a Wiener measurable functional of $\left(y_{1}, \cdots, y_{n}\right)$, then

$$
g\left[\sqrt{\frac{2}{t_{1}-t_{0}}} x\left(\cdot, t_{1}\right), \cdots, \sqrt{\frac{2}{t_{n}-t_{n-1}}}\left(x\left(\cdot, t_{n}\right)-x\left(\cdot, t_{n-1}\right)\right)\right]
$$

is a Yeh-Wiener measurable functional on $C_{2}[R]$ and

$$
\begin{aligned}
& \int_{C_{2}[R]} g\left[\sqrt{\frac{2}{t_{1}-t_{0}}} x\left(\cdot, t_{1}\right), \cdots, \sqrt{\frac{2}{t_{n}-t_{n-1}}}\left(x\left(\cdot, t_{n}\right)-x\left(\cdot, t_{n-1}\right)\right)\right] d x \\
= & \int_{C_{1}[a, b]} \cdots \int_{C_{1}[a, b]} g\left(y_{1}, \cdots, y_{n}\right) d y_{1} \cdots d y_{n} .
\end{aligned}
$$

Case I. Let $g\left(y_{1}, \cdots, y_{n}\right)=\chi_{I}\left(y_{1}, \cdots, y_{n}\right)$, where $I$ is the interval

${ }^{2}$ The proof has to proceed in the opposite order from the motivation because of the measurability argument. 
$I=\left\{\left(y_{1}, \cdots, y_{n}\right) \in \stackrel{n}{X}^{n} C_{1}[a, b] \mid-\infty \leqq z_{j, k}<y_{k}\left(s_{j}\right) \leqq w_{j, k} \leqq+\infty\right.$, for $j=$ $1, \cdots, m, k=1, \cdots, n\}$. Clearly $I=I_{1} \cap I_{2} \cap \cdots \cap I_{m}$ where $I_{j}=$ $\left\{\left(y_{1}, \cdots, y_{n}\right) \in \stackrel{n}{\times} C_{1}[a, b] \mid-\infty \leqq z_{j, k}<y_{k}\left(s_{j}\right) \leqq w_{j, k} \leqq+\infty\right.$, for $k=$ $1, \cdots, n\}$. Now

$$
\begin{aligned}
g[ & \left.\sqrt{\frac{2}{t_{1}-t_{0}}} x\left(\cdot, t_{1}\right), \cdots, \sqrt{\frac{2}{t_{n}-t_{n-1}}}\left(x\left(\cdot, t_{n}\right)-x\left(\cdot, t_{n-1}\right)\right)\right] \\
& =\prod_{j=1}^{m} \chi_{I_{j}}\left[\sqrt{\left.\frac{2}{t_{1}-t_{0}} x\left(\cdot, t_{1}\right), \cdots, \sqrt{\frac{2}{t_{n}-t_{n-1}}}\left(x\left(\cdot, t_{n}\right)-x\left(\cdot, t_{n-1}\right)\right)\right]}\right. \\
& =\prod_{j=1}^{m} \chi_{k_{j}}(x(\cdot, \cdot))
\end{aligned}
$$

where

$$
\begin{aligned}
K_{j} & =\left\{x \in C_{2}[R] \mid-\infty \leqq \sqrt{\frac{t_{k}-t_{k-1}}{2}} z_{j, k}<\left[x\left(s_{j}, t_{k}\right)-x\left(s_{j}, t_{k-1}\right)\right]\right. \\
& \left.\leqq \sqrt{\frac{t_{k}-t_{k-1}}{2}} w_{j, k} \leqq+\infty \text { for } k=1, \cdots, n\right\}
\end{aligned}
$$

and

$$
\chi_{K_{j}}(x(\cdot, \cdot))=\chi_{L_{j}}\left[x\left(s_{j}, t_{1}\right), \cdots, x\left(s_{j}, t_{n}\right)\right],
$$

where

$$
\begin{aligned}
L_{j} & =\left\{\left[u_{j, 1}, \cdots, u_{j, n}\right] \in R^{n} \mid-\infty \leqq \sqrt{\frac{t_{k}-t_{k-1}}{2}} z_{j, k}<u_{j, k}-u_{j, k-1}\right. \\
& \left.\leqq \sqrt{\frac{t_{k}-t_{k-1}}{2}} w_{j, k} \leqq+\infty \quad \text { for } \quad k=1, \cdots, n\right\} .
\end{aligned}
$$

Thus in this case (2.8) is Yeh-Wiener measurable on $C_{2}[R]$ since $\chi_{L_{j}}$ is a Lebesgue measurable function in $R^{m n}$. Integrating the expression (2.8) we obtain by using (2.10) and (2.11),

$$
\begin{aligned}
& G \equiv \int_{C_{2}[R]} g\left[\sqrt{\frac{2}{t_{1}-t_{0}}} x\left(\cdot, t_{1}\right), \cdots, \sqrt{\frac{2}{t_{n}-t_{n-1}}}\left(x\left(\cdot, t_{n}\right)-x\left(\cdot, t_{n-1}\right)\right)\right] d x \\
& =\int_{C_{2}[R]} \prod_{j=1}^{m} \chi_{L_{j}}\left[x\left(s_{j}, t_{1}\right), \cdots, x\left(s_{j}, t_{n}\right)\right] d x \\
& =\pi^{-(m n) / 2}\left[\left(s_{1}-s_{0}\right) \cdots\left(s_{m}-s_{m-1}\right)\right]^{-n / 2}\left[\left(t_{1}-t_{0}\right) \cdots\left(t_{n}-t_{n-1}\right)\right]^{-m / 2} \\
& \cdot \int_{-\infty}^{\infty} \cdots \int_{-\infty}^{(m n)} \prod_{j=1}^{m} \chi_{L_{j}}\left[u_{j, 1}, \cdots, u_{j, n}\right] \\
& \cdot \exp \left\{-\sum_{j=1}^{m} \sum_{k=1}^{n} \frac{\left(u_{j, k}-u_{j, k-1}-u_{j-1, k}+u_{j-1, k-1}\right)^{2}}{\left(s_{j}-s_{j-1}\right)\left(t_{k}-t_{k-1}\right)}\right\} d U \text {, }
\end{aligned}
$$

where $u_{j, 0} \equiv u_{0, k} \equiv 0$. If we set $v_{j, k}=\sqrt{2 /\left(t_{k}-t_{k-1}\right)}\left(u_{j, k}-u_{j, k-1}\right)$ so that $u_{j, k}=\sum_{i=1}^{k} \sqrt{\left(t_{i}-t_{i-1}\right) / 2} v_{j, i}$, we obtain 


$$
\begin{aligned}
G \equiv & (2 \pi)^{-(m n) / 2}\left[\left(s_{1}-s_{0}\right) \cdots\left(s_{m}-s_{m-1}\right)\right]^{-n / 2} \\
& \cdot \int_{-\infty}^{\infty} \cdots \int_{-\infty}^{(m n)} \prod_{j=1}^{m} \chi_{L_{j}}\left(\sqrt{\frac{t_{1}-t_{0}}{2}} v_{j, 1}, \cdots, \sum_{i=1}^{k} \sqrt{\frac{t_{i}-t_{i-1}}{2}} v_{j, i}, \cdots,\right. \\
& \left.\cdot \sum_{i=1}^{n} \sqrt{\frac{t_{i}-t_{i-1}}{2}} v_{j, i}\right) \exp \left\{-\sum_{j=1}^{m} \sum_{k=1}^{n} \frac{\left(v_{j, k}-v_{j-1, k}\right)^{2}}{2\left(s_{j}-s_{j-1}\right)}\right\} d v_{1,1} \cdots d v_{m, n} \\
= & \int_{C_{1}[a, b]} \cdots \int_{C_{1}[a, b]} \prod_{j=1}^{m} \chi_{I_{1}}\left(y_{1}\left(s_{j}\right), \cdots, y_{i}\left(s_{j}\right), \cdots, y_{n}\left(s_{j}\right)\right) d y_{1} \cdots d y_{n} \\
= & \int_{C_{1}[a, b]} \quad \cdots \int_{C_{1}[a, b]} g\left(y_{1}, \cdots, y_{n}\right) d y_{1} \cdots d y_{n}
\end{aligned}
$$

and Case $I$ is proved. The remaining cases are analogous to those of Theorem 1 and are proved in the same way.

3. The orthogonal transformation. Theorem 2 which we have just proved gives us an evaluation of the Yeh-Wiener integral of a functional $F(x(\cdot, \cdot))$ which depends only on the values of $x$ on $n$ parallel lines. It is natural to inquire next concerning functionals that depend solely on the values of $x$ on two perpendicular lines. We shall limit our investigation in this paper to the case where the two perpendicular lines are the free edges of the fundamental rectangle. Before we can obtain such a theorem, we will need to establish a generalization of Bearman's theorem [1,130] on rotations in the product of two Wiener spaces. (A theorem of this sort was once proved by Edwin Sheffield, but so far as the authors know, it was never published.)

Theorem 3. Let $F\left(y_{1}, \cdots, y_{n}\right)$ be any Wiener integrable functional of $y_{1}(\cdot), \cdots, y_{n}(\cdot)$ on $\stackrel{n}{\times} C_{1}[a, b]$ and let $\left(c_{i, j}\right)_{i, j=1}, \ldots, n$ be a real orthogonal matrix (so that $\sum_{k=1}^{n} c_{i, k} c_{j, k}=\delta_{i, j}$ for $i, j=1, \cdots, n$ ). Then the transformation

$$
y_{i}(\cdot)=\sum_{j=1}^{n} c_{i, j} z_{j}(\cdot) \quad \text { for } \quad i=1, \cdots, n
$$

is a measure preserving transformation of $\stackrel{n}{\times} C_{1}[a, b]$ onto itself. Moreover,

$$
\begin{aligned}
& \int_{C_{1}[a, b]} \cdots \int_{C_{1}[a, b]} F\left(y_{1}, \cdots, y_{n}\right) d y_{1} \cdots d y_{n} \\
= & \int_{C_{1}[a, b]} \cdots \int_{C_{1}[a, b]} F\left(\sum_{j=1}^{n} c_{1, j} z_{j}, \cdots, \sum_{j=1}^{n} c_{n, j} z_{j}\right) d z_{1} \cdots d z_{n} .
\end{aligned}
$$

Proof. Case I. Let $F$ depend only on the values of $y_{1}, \cdots, y_{n}$ at a certain finite set of points, $a=s_{0}<s_{1}<\cdots<s_{m}=b$, i.e., let 


$$
\begin{aligned}
& F\left(y_{1}, \cdots y_{n}\right)=f\left(y_{1}\left(s_{1}\right), \cdots y_{1}\left(s_{m}\right) ; y_{2}\left(s_{1}\right), \cdots,\right. \\
& \left.y_{2}\left(s_{m}\right) ; \cdots ; y_{n}\left(s_{1}\right), \cdots, y_{n}\left(s_{m}\right)\right),
\end{aligned}
$$

where $f\left(u_{1,1}, \cdots u_{1, m} ; \cdots ; u_{n, 1}, \cdots, u_{n, m}\right)$ is a bounded measurable function of its $n m$ arguments. It is clear that $F$ is Wiener measurable and bounded on $\stackrel{n}{\times} C_{1}[a, b]$. Now we have

$$
\begin{aligned}
I \equiv & \int_{C_{1}[a, b]} \cdots \int_{C_{1}[a, b]} F\left(y_{1}, \cdots, y_{n}\right) d y_{1} \cdots d y_{n} \\
(3.3)= & (2 \pi)^{-(m n) / 2}\left[\left(s_{1}-a\right) \cdots\left(s_{m}-s_{m-1}\right)\right]^{-n / 2} \int_{-\infty}^{\infty} \cdots \int_{-\infty}^{(m n)} \\
& \cdot f\left(u_{1,1}, \cdots, u_{n, m}\right) \exp \left\{-\sum_{i=1}^{n} \sum_{k=1}^{m} \frac{\left(u_{i, k}-u_{i, k-1}\right)^{2}}{2\left(s_{k}-s_{k-1}\right)}\right\} d u_{1,1} \cdots d u_{n, m},
\end{aligned}
$$

where $u_{i, 0} \equiv 0$.

Let us make the transformation $u_{i, k}=\sum_{j=1}^{n} c_{i, j} v_{j, k}$ where $i=$ $1, \cdots, n$ and $k=1, \cdots, m$, to obtain

$$
\begin{aligned}
& I=(2 \pi)^{-(m n) / 2}\left[\left(s_{1}-a\right) \cdots\left(s_{m}-s_{m-1}\right)\right]^{-n / 2} \int_{-\infty}^{\infty} \cdots \int_{-\infty}^{\infty} \\
& \cdot f\left(\sum_{j=1}^{n} c_{1, j} v_{j, 1}, \cdots, \sum_{j=1}^{n} c_{n, j} v_{j, m}\right) \\
& \cdot \exp \left\{-\sum_{k=1}^{m} \sum_{i=1}^{n} \frac{\left(\sum_{j=1}^{n} c_{i, j}\left(v_{j, k}-v_{j, k-1}\right)\right)^{2}}{2\left(s_{k}-s_{k-1}\right)}\right\} d v_{1,1} \cdots d v_{n, m} .
\end{aligned}
$$

Since $\left(c_{i, j}\right)$ is an orthogonal matrix,

$$
\sum_{i=1}^{n}\left(\sum_{j=1}^{n} c_{i, j}\left(v_{j, k}-v_{j, k-1}\right)\right)^{2}=\sum_{j=1}^{n}\left(v_{j, k}-v_{j, k-1}\right)^{2}
$$

and we obtain

$$
\begin{aligned}
I= & (2 \pi)^{-(m n) / 2}\left[\left(s_{1}-a\right) \cdots\left(s_{m}-s_{m-1}\right)\right]^{-n / 2} \int_{-\infty}^{\infty} \cdots \int_{-\infty}^{\infty} \\
& \cdot f\left(\sum_{j=1}^{n} c_{1, j} v_{j, 1}, \cdots, \sum_{j=1}^{n} c_{n, j} v_{j, m}\right) \exp \left\{-\sum_{k=1}^{m} \sum_{j=1}^{n} \frac{\left(v_{j, k}-v_{j, k-1}\right)^{2}}{2\left(s_{k}-s_{k-1}\right)}\right\} \\
& \cdot d v_{1,1} \cdots d v_{n, m} \\
= & \int_{C_{1}[a, b]} \cdots \int_{C_{1}[a, b]} f\left(\sum_{j=1}^{n} c_{1, j} z_{j}\left(s_{1}\right), \cdots, \sum_{j=1}^{n} c_{n, j} z_{j}\left(s_{m}\right)\right) d z_{1} \cdots d z_{n} \\
= & \int_{C_{1}[a, b]} \cdots \int_{C_{1}[a, b]} F\left(\sum_{j=1}^{n} c_{1, j} z_{j}(\cdot), \cdots, \sum_{j=1}^{n} c_{n, j} z_{j}(\cdot)\right) d z_{1} \cdots d z_{n} .
\end{aligned}
$$

In the above argument, the measurability of each successive integrand follows from the measurability of $f\left(u_{1,1}, \cdots, u_{n, m}\right)$, and the boundedness of $f$ implies the integrability of each integrand. Thus (3.1) is established for Case I. If we apply (3.1) to the case where $f$ is a characteristic function of a measurable set we observe that (3.0) is a measure preserving transformation of $\stackrel{n}{\times}^{\prime} C_{1}[a, b]$ onto itself. 
Case II. Let $F\left(y_{1}, \cdots, y_{n}\right)=\chi_{\Omega}\left(y_{1}, \cdots, y_{n}\right)$, where $\Omega$ is the union of a countable disjoint set of intervals $\Omega=\bigcup_{j=1}^{\infty} I_{j}$ and each $I_{j}$ is an interval in the product space $\stackrel{n}{\times}^{\prime} C_{1}[a, b]$, (as in the proof of Theorem 2, Case I). Because each $\chi_{I_{j}}$ satisfies the hypothesis of Case I, the theorem holds when $F$ is of the form $F\left(y_{1}, \cdots, y_{n}\right)=$ $\chi_{I_{j}}\left(y_{1}, \cdots, y_{n}\right)$. Since $\Omega$ is the countable union of measurable sets, it is measurable, and by summing both sides of (3.1) applied to $\chi_{I_{j}}$ we obtain (3.1) applied to $\chi_{\Omega}$.

Case III. Let $F=\chi_{E}\left(y_{1}, \cdots, y_{n}\right)$ where $E$ is a Wiener measurable set in $X^{n} C_{1}[a, b]$. The result of Case II can be extended from $\Omega=$ $\bigcup_{j=1}^{\infty} I_{j}$ to countable intersections of sets of this form and then to null sets and then to general measurable sets in the usual way.

Case IV. Let $F$ be a nonnegative functional. If $F$ is actually a simple functional the result follows from Case III by multiplication by constants and addition. If $F$ is not a simple functional, it can be expressed as a limit of a monotone increasing sequence of simple functionals, and the theorem follows for this case.

Case V. General Case: If $F$ is real, we write $F=F^{+}-F^{-}$ and apply Case IV to $F^{+}$and to $F^{-}$and thus establish the theorem for real functionals. The extension to complex functionals is immediate.

4. The two perpendicular lines theorem. We now proceed to establish a formula for the evaluation of the Yeh-Wiener integral of a functional that depends solely on the values of $x$ on two perpendicular lines.

THEOREM 4. Let $f(z, y)$ defined on $C_{1}[a, b] \times C_{1}[\alpha, \beta]$ be a functional such that

$$
f^{\prime}\left(\sqrt{\frac{\beta-\alpha}{2}} z,[(\cdot)-\alpha]\left[\sqrt{\frac{b-a}{2}} \int_{(\cdot)}^{\beta} \frac{d y(\tau)}{\tau-\alpha}+\frac{z(b)}{\sqrt{2(\beta-\alpha)}}\right]\right)
$$

is Wiener measurable on $C_{1}[a, b] \times C_{1}[\alpha, \beta]$. Then it follows that $f[x(\cdot, \beta), x(b, \cdot)]$ is Yeh-Wiener measurable on $C_{2}[R]$, where $R \equiv$ $[a, b] \times[\alpha, \beta]$. Moreover,

$$
\begin{aligned}
& \int_{C_{2}[R]} f[x(\cdot, \beta), x(b, \cdot)] d x \\
= & \int_{C_{1}[a, b] \times C_{1}[\alpha, \beta]} f\left\{\sqrt{\frac{\beta-\alpha}{2}} z,[(\cdot)-\alpha]\left[\sqrt{\frac{b-\alpha}{2}} \int_{(\cdot)}^{\beta} \frac{d y(\tau)}{\tau-\alpha}\right.\right. \\
& \left.\left.+\frac{z(b)}{\sqrt{2(\beta-\alpha)}}\right]\right\} d(z \times y)
\end{aligned}
$$


where the existence of either member implies the existence of the other and their equality.

Proof ${ }^{3} \quad$ Case I. $\quad$ Let $f(z, y)=g\left(z ; y\left(t_{1}\right), \cdots, y\left(t_{n}\right)\right)$, where $\alpha=t_{0}<$ $t_{1}<\cdots<t_{n}=\beta$ and let $g\left(z ; u_{1}, \cdots, u_{n}\right)$ be the characteristic functional of a half-open interval $I$ in $C_{1}[a, b] \times R^{n}$; i.e., $I=\left\{\left(z ; u_{1}, \cdots, u_{n}\right) \mid-\infty \leqq\right.$ $\gamma_{j}<z\left(s_{j}\right) \leqq \delta_{j} \leqq+\infty$ for $j=1, \cdots, m ;-\infty \leqq c_{k}<u_{k} \leqq d_{k} \leqq+\infty$ for $k=1, \cdots, n\}, a=s_{0}<s_{1}<\cdots<s_{m}=b$.

The right member of (4.1) becomes

$$
\begin{aligned}
& I_{2} \equiv \int_{C_{1}[a, b]} \int_{C_{1}[\alpha, \beta]} g\left\{\sqrt{\frac{\beta-\alpha}{2}} z ;\left(t_{1}-\alpha\right)\right. \\
& \cdot\left[\sqrt{\frac{b-\alpha}{2}} \int_{t_{1}}^{\beta} \frac{d y(\tau)}{\tau-\alpha}+\frac{z(b)}{\sqrt{2(\beta-\alpha)}}\right], \cdots, \\
&\left(t_{n-1}-\alpha\right)\left[\sqrt{\frac{b-\alpha}{2}} \int_{t_{n-1}}^{\beta} \frac{d y(\tau)}{\tau-\alpha}+\frac{z(b)}{\sqrt{2(\beta-\alpha)}}\right], \\
&\left.\left(t_{n}-\alpha\right) \frac{z(b)}{\sqrt{2(\beta-\alpha)}}\right\} d y d z .
\end{aligned}
$$

We now apply the well-known result: If $\varphi_{1}, \cdots, \varphi_{n}$ are orthonormal on $[a, b]$ and of bounded variation on $[a, b]$ and if $h\left(u_{1}, \cdots, u_{n}\right)$ is measurable, then

$$
\begin{aligned}
& \int_{C_{1}[a, b]} h\left\{\int_{a}^{b} \varphi_{1}(t) d x(t), \cdots, \int_{a}^{b} \varphi_{n}(t) d x(t)\right\} d x \\
= & (2 \pi)^{-n / 2} \int_{-\infty}^{\infty} \cdots \int_{-\infty}^{\infty} h\left(u_{1}, \cdots, u_{n}\right) \exp \left\{-\sum_{j=1}^{n} \frac{u_{j}^{2}}{2}\right\} d u_{1} \cdots d u_{n},
\end{aligned}
$$

where the existence of either member implies that of the other and their equality.

To apply this result, we let

$$
\theta_{j}(t)=\left\{\begin{array}{ccc}
0 & \text { if } \quad \alpha \leqq t \leqq t_{j}, \\
\frac{1}{t-\alpha} & \text { if } \quad t_{j}<t \leqq \beta
\end{array} \quad \text { for } \quad j=1, \cdots, n\right.
$$

and note that $\theta_{n}(t) \equiv 0$, so

$$
\int_{t_{j}}^{\beta} \frac{d y(\tau)}{\tau-\alpha}=\int_{\alpha}^{\beta} \theta_{j}(t) d y(t), \quad \text { for } \quad j=1, \cdots, n .
$$

We define

3 This proof is given in the logical order. For motivation read in reverse order, using the inverse of the matrix $\left(c_{k, j}\right)$. 


$$
\begin{aligned}
\varphi_{j}(t) & =\frac{-\left[\theta_{j+1}(t)-\theta_{j}(t)\right]}{\sqrt{\int_{\alpha}^{\beta}\left(\theta_{j+1}(\tau)-\theta_{j}(\tau)\right)^{2} d \tau}} \\
& =-\sqrt{\frac{\left(t_{j}-\alpha\right)\left(t_{j+1}-\alpha\right)}{t_{j+1}-t_{j}}}\left[\theta_{j+1}(t)-\theta_{j}(t)\right] \text { for } j=1, \cdots, n-1,
\end{aligned}
$$

and observe that $\left\{\varphi_{1}, \cdots, \varphi_{n-1}\right\}$ forms an orthonormal set on $[\alpha, \beta]$. To solve for $\theta_{j}$ we write

$$
\theta_{j+1}(t)-\theta_{j}(t)=-\sqrt{\frac{t_{j+1}-t_{j}}{\left(t_{j}-\alpha\right)\left(t_{j+1}-\alpha\right)}} \varphi_{j}(t)
$$

and sum from $j=k$ to $j=n-1$ to obtain

$$
\theta_{k}(t)=\sum_{j=k}^{n-1} \sqrt{\frac{t_{j+1}-t_{j}}{\left(t_{j}-\alpha\right)\left(t_{j+1}-\alpha\right)}} \varphi_{j}(t)
$$

and consequently (4.4) becomes

$$
\int_{t_{k}}^{\beta} \frac{d y(\tau)}{\tau-\alpha}=\sum_{j=k}^{n-1} \sqrt{\frac{t_{j+1}-t_{j}}{\left(t_{j}-\alpha\right)\left(t_{j+1}-\alpha\right)}} \int_{\alpha}^{\beta} \varphi_{j}(t) d y(t)
$$

Substituting the value of $\int_{t_{k}}^{\beta}(d y(\tau)) /(\tau-\alpha)$ for $k=1, \cdots, n-1$ into (4.2) we obtain

$$
\begin{aligned}
I_{2}= & \int_{C_{1}[a, b]} \int_{C_{1}[\alpha, \beta]} g\left\{\sqrt{\frac{\beta-\alpha}{2}} z ;\left(t_{1}-\alpha\right)\left[\sqrt{\frac{b-a}{2}}\right.\right. \\
& \left.\cdot \sum_{j=1}^{n-1} \sqrt{\frac{t_{j+1}-t_{j}}{\left(t_{j}-\alpha\right)\left(t_{j+1}-\alpha\right)}} \int_{\alpha}^{\beta} \varphi_{j}(t) d y(t)+\frac{z(b)}{\sqrt{2(\beta-\alpha)}}\right], \cdots, \\
& \left(t_{n-1}-\alpha\right)\left[\sqrt{\frac{b-a}{2}} \sqrt{\frac{t_{n}-t_{n-1}}{\left(t_{n-1}-\alpha\right)\left(t_{n}-\alpha\right)}} \int_{\alpha}^{\beta} \varphi_{n-1}(t) d y(t)\right. \\
& \left.\left.+\frac{z(b)}{\sqrt{2(\beta-\alpha)}}\right], \quad\left(t_{n}-\alpha\right) \frac{z(b)}{\sqrt{2(\beta-\alpha)}}\right\} d y d z .
\end{aligned}
$$

We now use (4.3) to evaluate the inner Wiener integral above and obtain

$$
\begin{aligned}
I_{2}= & \int_{C_{1}[a, b]}(2 \pi)^{-(n-1) / 2} \int_{-\infty}^{\infty} \cdots \int_{-\infty}^{\infty} g\left\{\sqrt{\frac{\beta-\alpha}{2}} z ;\right. \\
& \left(t_{1}-\alpha\right)\left[\frac{z(b)}{\sqrt{2(\beta-\alpha)}}+\sqrt{\frac{b-a}{2}} \sum_{j=1}^{n-1} \sqrt{\frac{t_{j+1}-t_{j}}{\left(t_{j}-\alpha\right)\left(t_{j+1}-\alpha\right)}} u_{j}\right], \cdots, \\
& \left(t_{n-1}-\alpha\right)\left[\frac{z(b)}{\sqrt{2(\beta-\alpha)}}+\sqrt{\frac{b-a}{2}} \sqrt{\frac{t_{n}-t_{n-1}}{\left(t_{n-1}-\alpha\right)\left(t_{n}-\alpha\right)}} u_{n-1}\right], \\
& \left.\left(t_{n}-\alpha\right) \frac{z(b)}{\sqrt{ } 2(\beta-\alpha)}\right\} \exp \left\{-\frac{u_{1}^{2}}{2}-\cdots-\frac{u_{n-1}^{2}}{2}\right\} d u_{1} \cdots d u_{n-1} d z .
\end{aligned}
$$


If we set $v_{j}=u_{j} \sqrt{b-a}$, then

$$
\begin{aligned}
I_{2}= & \int_{C_{1}[a, b]}(2 \pi(b-a))^{-((n-1) / 2)} \int_{-\infty}^{\infty} \cdots \int_{-\infty}^{(n-1)} g\left\{\sqrt{\frac{\beta-\alpha}{2}} z ;\right. \\
& \left(t_{1}-\alpha\right)\left[\frac{z(b)}{\sqrt{2(\beta-\alpha)}}+\frac{1}{\sqrt{2}} \sum_{j=1}^{n-1} \sqrt{\frac{t_{j+1}-t_{j}}{\left(t_{j}-\alpha\right)\left(t_{j+1}-\alpha\right)}} v_{j}\right], \cdots, \\
& \left(t_{n-1}-\alpha\right)\left[\frac{z(b)}{\sqrt{2(\beta-\alpha)}}+\frac{1}{\sqrt{2}} \sqrt{\left.\frac{t_{n}-t_{n-1}}{\left(t_{n-1}-\alpha\right)\left(t_{n}-\alpha\right)} v_{n-1}\right],}\right. \\
& \left.\left(t_{n}-\alpha\right) \frac{z(b)}{\sqrt{2(\beta-\alpha)}}\right\} \exp \left\{-\frac{v_{1}^{2}}{2(b-a)}-\cdots-\frac{v_{n-1}^{2}}{2(b-a)}\right\} \\
& . d v_{1} \cdots d v_{n-1} d z .
\end{aligned}
$$

Using the formula

$$
\frac{1}{\sqrt{2 \pi(b-a)}} \int_{-\infty}^{\infty} F(v) e^{-v^{2} /(2(b-a))} d v=\int_{C_{1}[a, b]} F(x(b)) d x
$$

$(n-1)$ times, we see that (replacing $z$ by $z_{n}$ )

$$
\begin{aligned}
I_{2}= & \int_{C_{1}[a, b]} \cdots \int_{C_{1}[a, b]} g\left\{\sqrt{\frac{\beta-\alpha}{2}} z_{n} ; \frac{t_{1}-\alpha}{\sqrt{2}}\left[\frac{z_{n}(b)}{\sqrt{\beta-\alpha}}\right.\right. \\
& \left.+\sum_{j=1}^{n-1} \sqrt{\frac{t_{j+1}-t_{j}}{\left(t_{j}-\alpha\right)\left(t_{j+1}-\alpha\right)}} z_{j}(b)\right], \cdots,\left(\frac{t_{n-1}-\alpha}{\sqrt{2}}\right)\left[\frac{z_{n}(b)}{\sqrt{\beta-\alpha}}\right. \\
& \left.\left.+\sqrt{\frac{t_{n}-t_{n-1}}{\left(t_{n-1}-\alpha\right)\left(t_{n}-\alpha\right)}} z_{n-1}(b)\right], \frac{\left(t_{n}-\alpha\right)}{\sqrt{2}} \frac{z_{n}(b)}{\sqrt{\beta-\alpha}}\right\} d z_{1} \cdots d z_{n} .
\end{aligned}
$$

We next apply Theorem 3, using the transformation

$$
z_{k}=\sum_{j=1}^{n} c_{k, j} y_{j}
$$

where for $k \leqq n-1$

and

$$
c_{k, j}= \begin{cases}\sqrt{\frac{\left(t_{k+1}-t_{k}\right)\left(t_{j}-t_{j-1}\right)}{\left(t_{k+1}-\alpha\right)\left(t_{k}-\alpha\right)}} & \text { if } j \leqq k \\ -\sqrt{\frac{t_{k}-\alpha}{t_{k+1}-\alpha}} & \text { if } j=k+1 \\ 0 & \text { if } j>k+1\end{cases}
$$

$$
c_{n, j}=\sqrt{\frac{t_{j}-t_{j-1}}{t_{n}-\alpha}} \quad \text { for } \quad j=1, \cdots, n .
$$

We note that $\left(c_{k, j}\right)$ forms a real orthogonal matrix and that

$$
\begin{aligned}
& \left(t_{k}-\alpha\right)\left[\frac{z_{n}}{\sqrt{\beta-\alpha}}+\sum_{j=k}^{n-1} \sqrt{\frac{t_{j+1}-t_{j}}{\left(t_{j}-\alpha\right)\left(t_{j+1}-\alpha\right)}} z_{j}\right] \\
= & \sum_{1}^{k} \sqrt{t_{j}-t_{j-1}} y_{j}, \text { for } k=1, \cdots, n .
\end{aligned}
$$


Thus by Theorem 3

$$
\begin{aligned}
I_{2}= & \int_{C_{1}[a, b]} \stackrel{(n)}{\cdots} \int_{C_{1}[a, b]} g\left\{\sum_{j=1}^{n} \sqrt{\frac{t_{j}-t_{j-1}}{2}} y_{j} ;\right. \\
& \left.\sqrt{\frac{t_{1}-\alpha}{2}} y_{1}(b), \cdots, \sum_{j=1}^{n} \sqrt{\frac{t_{j}-t_{j-1}}{2}} y_{j}(b)\right\} d y_{1} \cdots d y_{n}
\end{aligned}
$$

and by Theorem 2,

$$
I_{2}=\int_{C_{2}[R]} g\left(x\left(\cdot, t_{n}\right) ; x\left(b, t_{1}\right), \cdots, x\left(b, t_{n}\right)\right) d x=\int_{C_{2}[R]} f[x(\cdot, \beta), x(b, \cdot)] d x,
$$

and Case I is established.

We then proceed as in Theorem 1 to establish Theorem 4.

5. Applications of Theorem 4 .

EXAMPLE 1. Let us apply Theorem 4 to the functional:

$$
f(z, y)=\int_{a}^{b} p(s)[z(s)]^{2} d s \int_{\alpha}^{\beta} q(t)[y(t)]^{2} d t
$$

where $p \in L_{1}[a, b]$ and $q \in L_{1}[\alpha, \beta]$. Then

$$
\begin{aligned}
I \equiv & \int_{C_{2}[R]}\left\{\int_{a}^{b} p(s)[x(s, \beta)]^{2} d s \int_{\alpha}^{\beta} q(t)[x(b, t)]^{2} d t\right\} d x \\
= & \int_{C_{1}[\alpha, b]} \int_{C_{1}[\alpha, \beta]} \int_{a}^{b} p(s)\left(\frac{\beta-\alpha}{2}\right) z^{2}(s) d s \int_{\alpha}^{\beta} q(t)\left[(t-\alpha)^{2}\right] \\
& \cdot\left[\sqrt{\frac{b-\alpha}{2}} \int_{t}^{\beta} \frac{d y(\tau)}{\tau-\alpha}+\frac{z(b)}{\sqrt{2(\beta-\alpha)}}\right]^{2} d t d y d z
\end{aligned}
$$

and each expression can be evaluated by known techniques to yield

$$
\begin{aligned}
I= & \frac{1}{4} \int_{a}^{b} \int_{\alpha}^{\beta} p(s) q(t)(s-a)(t-\alpha)[(b-a)(\beta-\alpha) \\
& +2(s-a)(t-\alpha)] d x d t .
\end{aligned}
$$

EXAMPle 2. We next show how to calculate the following integral using Theorem 4: (the authors know of no way of evaluating the integral without applying Theorem 4)

$$
I \equiv \int_{C_{2}[R]} \exp \left\{A \int_{a}^{b}[x(s, \beta)]^{2} d s+B \int_{\alpha}^{\beta} x(b, t) d t\right\} d x
$$

Let us set

$$
f(z, y)=\exp \left\{A \int_{a}^{b}[z(s)]^{2} d s+B \int_{\alpha}^{\beta} y(t) d t\right\}
$$


By Theorem 4,

$$
\begin{aligned}
I= & \int_{C_{1}[a, b]} \int_{C_{1}[\alpha, \beta]} \exp \left\{\frac{A(\beta-\alpha)}{2} \int_{a}^{b}[z(s)]^{2} d s\right. \\
& \left.+B \int_{\alpha}^{\beta}[t-\alpha]\left[\sqrt{\frac{b-\alpha}{2}} \int_{t}^{\beta} \frac{d y(\tau)}{\tau-\alpha}+\frac{z(b)}{\sqrt{2(\beta-\alpha)}}\right] d t\right\} d y d z \\
= & I_{1} \cdot I_{2} \text { where }
\end{aligned}
$$

(5.6) $\quad I_{1}=\int_{C_{1}[a, b]} \exp \left\{\frac{A(\beta-\alpha)}{2} \int_{a}^{b}[z(s)]^{2} d s\right\} \exp \left\{B \int_{\alpha}^{\beta} \frac{(t-\alpha) z(b)}{\sqrt{2(\beta-\alpha)}} d t\right\} d z$

and

$$
I_{2}=\int_{C_{1}[\alpha, \beta]} \exp \left\{B \sqrt{\frac{b-a}{2}} \int_{\alpha}^{\beta}(t-\alpha) \int_{t}^{\beta} \frac{d y(\tau)}{\tau-\alpha} d t\right\} d y
$$

To evaluate $I_{1}$, we shall use the following theorem of Cameron and Martin [2, 75] where we have changed the scale and the variance:

TheOREm 1a. Let $q(t)$ be continuous and positive on $[a, b]$ and let $\mu_{0}$ be the least characteristic value of the differential equation

$$
h^{\prime \prime}(s)+\mu q(s) h(s)=0
$$

subject to the boundary conditions

$$
h(a)=h^{\prime}(b)=0 .
$$

Then if $F(x)$ is any Wiener measurable functional, if $\mu<\mu_{0}$, and if $h_{\mu}(t)$ is any nontrivial solution of $(5.8)$ satisfying $h_{\mu}^{\prime}(b)=0$, we have

$$
\begin{aligned}
& \int_{C_{1}[a, b]} F(x) \exp \left\{\frac{\mu}{2} \int_{a}^{b} q(s) x^{2}(s) d s\right\} d x \\
& =\sqrt{\frac{h_{\mu}(b)}{h_{\mu}(a)}} \int_{C_{1}[a, b]} F\left[h_{\mu}(\cdot) \int_{a}^{(\cdot)} \frac{d y(\sigma)}{h_{\mu}(\sigma)}\right] d y
\end{aligned}
$$

where the existence of either member implies that of the other and their equality.

We now identify in the expression for $I_{1}$ in (5.6)

$$
F(z)=\exp \left\{\frac{B z(b)}{\sqrt{2(\beta-\alpha)}} \int_{\alpha}^{\beta}(t-\alpha) d t\right\}=\exp \left\{B z(b)\left(\frac{\beta-\alpha}{2}\right)^{3 / 2}\right\} .
$$

Let $q(s) \equiv 1, \mu=A(\beta-\alpha)$. An examination of the differential system shows that the least characteristic value is $\mu_{0}=\pi^{2} /\left(4(b-a)^{2}\right)$. We must therefore have $A<\pi^{2}\left[4(b-a)^{2}(\beta-\alpha)\right]^{-1}$. Now 


$$
\begin{aligned}
h_{\mu}(s) & =\cos \left((s-b) \mu^{1 / 2}\right) \\
& =\cos ((s-b) \sqrt{A(\beta-\alpha)}),
\end{aligned}
$$

and our integral $I_{1}$ may be evaluated:

$$
\begin{aligned}
I_{1}= & \sqrt{\frac{1}{\cos ((b-\alpha) \sqrt{A(\beta-\alpha))}}} \int_{C_{1}[a, b]} \\
& \cdot \exp \left\{B\left(\frac{\beta-\alpha}{2}\right)^{3 / 2} \int_{a}^{b} \frac{d y(\sigma)}{\cos ((\sigma-b) \sqrt{A(\beta-\alpha))}}\right\} d y .
\end{aligned}
$$

In order to employ (4.3), we normalize the secant function appearing in the Stieltjes integral, i.e., since

$$
\int_{a}^{b} \sec ^{2}[(\sigma-b) \sqrt{A(\beta-\alpha)}] d \sigma=\frac{\tan [(b-a) \sqrt{A(\beta-\alpha)]}}{\sqrt{A(\beta-\alpha)}}
$$

we let $p(\sigma)=\sec [(\sigma-b) \sqrt{A(\beta-\alpha)}](\tan \gamma)^{-1 / 2}[A(\beta-\alpha)]^{1 / 4}$, where $\gamma=$ $(b-a) \sqrt{A(\beta-\alpha)}$. Our integral $I_{1}$ becomes

$$
I_{1}=\sqrt{\sec \gamma} \int_{C_{1}[a, b]} \exp \left\{c \cdot \int_{a}^{b} p(\sigma) d y(\sigma)\right\} d y
$$

where $c=B((\beta-\alpha) / 2)^{3 / 2} \sqrt{\tan \gamma}[A(\beta-\alpha)]^{-(1 / 4)}$.

We apply (4.3) to obtain

$$
\begin{aligned}
I_{1}= & \sqrt{\frac{\sec \gamma}{2 \pi}} \int_{-\infty}^{\infty} e^{c u} e^{-u^{2} / 2} d u \\
= & \sqrt{\sec \gamma} \exp \left\{\frac{B^{2}\left(\frac{\beta-\alpha}{2}\right)^{3} \tan \gamma(A(\beta-\alpha))^{-1 / 2}}{2}\right\} \\
= & {\left[\sec \left[(b-a)(A(\beta-\alpha))^{1 / 2}\right]\right]^{1 / 2} } \\
& \cdot \exp \left\{\frac{B^{2}(\beta-\alpha)^{5 / 2} \tan \left[(b-\alpha)[A(\beta-\alpha)]^{1 / 2}\right]}{A^{1 / 2} 2^{4}}\right\} .
\end{aligned}
$$

In $I_{2}$, we set

$$
\begin{aligned}
J & =\int_{\alpha}^{\beta}(t-\alpha) \int_{t}^{\beta} \frac{d y(\tau)}{\tau-\alpha} d t \\
& =\int_{\alpha}^{\beta}\left(\int_{\alpha}^{\tau}(t-\alpha) d t\right) \frac{d y(\tau)}{\tau-\alpha} \\
& =\frac{1}{2} \int_{\alpha}^{\beta}(\tau-\alpha) d y(\tau)
\end{aligned}
$$

We normalize the integrand of this Stieltjes integral and set $p(\tau)=$ $(\tau-\alpha)(\beta-\alpha)^{-3 / 2} \sqrt{3}$, so the integral becomes 


$$
J=\frac{(\beta-\alpha)^{3 / 2}}{2 \sqrt{3}} \int_{\alpha}^{\beta} p(\tau) d y(\tau)
$$

and (5.7) becomes

$$
\begin{aligned}
I_{2} & =\int_{C_{1}[a, b]} \exp \left\{\frac{B \sqrt{b-\alpha}(\beta-\alpha)^{3 / 2}}{2 \sqrt{2} \sqrt{3}} \int_{\alpha}^{\beta} p(\tau) d y(\tau)\right\} d y \\
& =\frac{1}{\sqrt{2 \pi}} \int_{-\infty}^{\infty} \exp \left\{\frac{B \sqrt{b-a}(\beta-\alpha)^{3 / 2} u}{2 \sqrt{6}}-\frac{u^{2}}{2}\right\} d u \\
& =\exp \left\{\frac{B^{2}(b-\alpha)(\beta-\alpha)^{3}}{3 \cdot 2^{4}}\right\} .
\end{aligned}
$$

Thus our original integral has the value $I=I_{1} \cdot I_{2}$, so that

$$
\begin{aligned}
I= & {\left[\sec \left[(b-a)(A(\beta-\alpha))^{1 / 2}\right]\right]^{1 / 2} } \\
& \cdot \exp \left\{\frac{B^{2}(\beta-\alpha)^{5 / 2} \tan \left[(b-a)(A(\beta-\alpha))^{1 / 2}\right]}{A^{1 / 2} 2^{4}}\right\} \\
& \cdot \exp \left\{\frac{B^{2}(b-a)(\beta-\alpha)^{3}}{3 \cdot 2^{4}}\right\},
\end{aligned}
$$

where $A<\pi^{2} /\left(4(b-a)^{2}(\beta-\alpha)\right)$ and $A \neq 0$.

6. General functionals. Finally we consider a class of functionals which are not required to depend only on the values of $x$ on a restricted set. We do this by approximating $F(x)$ by a sequence of functionals $F\left(x_{n}\right)$ where $x_{n}$ is determined by the values of $x$ on $n$ horizontal lines and is defined in between the lines by linear interpolation. We then apply Theorem 2 to $F\left(x_{n}\right)$ and take limits.

THEOREM 5. Let $F(x)$ be a functional which is bounded and continuous in the uniform topology on $C_{2}[R]$. Let

$$
g_{\sigma}\left[y_{1}, \cdots, y_{n} ; s, t\right]=\left(\frac{t_{k}-t}{t_{k}-t_{k-1}}\right) y_{k-1}(s)+\left(\frac{t-t_{k-1}}{t_{k}-t_{k-1}}\right) y_{k}(s)
$$

for $a \leqq s \leqq b, t_{k-1} \leqq t \leqq t_{k}, y_{k} \in C_{1}[a, b]$ for $k=1, \cdots, n$; where $\sigma$ is $a$ subdivision, $\alpha=t_{0}<t_{1}<\cdots<t_{n}=\beta$, and norm $\sigma=\max _{k=1, \cdots, n}\left|t_{k}-t_{k-1}\right|$, and $y_{0} \equiv \mathbf{0}$.

Then

$$
\begin{aligned}
& \int_{C_{2}[R]} F(x) d x=\lim _{\text {norm } \sigma \rightarrow 0} \int_{C_{1}[a, b]} \stackrel{(n)}{\cdots} \int_{C_{1}[a, b]} F\left\{g _ { a } \left[\sqrt{\frac{t_{1}-t_{0}}{2}} y_{1}, \cdots,\right.\right. \\
& \left.\left.\sqrt{\frac{t_{1}-t_{0}}{2}} y_{1}+\cdots+\sqrt{\frac{t_{n}-t_{n-1}}{2}} y_{n}\right]\right\} d y_{1} \cdots d y_{n} .
\end{aligned}
$$

Proof. If we set 


$$
f\left(y_{1}, \cdots, y_{n}\right)=F\left\{g_{\sigma}\left[y_{1}, \cdots, y_{n} ; \cdot, \cdot\right]\right\}
$$

so that

$$
f\left[x\left(\cdot, t_{1}\right), \cdots, x\left(\cdot, t_{n}\right)\right]=F\left\{g_{\sigma}\left[x\left(\cdot, t_{1}\right), \cdots, x\left(\cdot, t_{n}\right) ; \cdot, \cdot\right]\right\},
$$

our functional $f$ satisfies the hypotheses of Theorem 2 and we have

$$
\begin{aligned}
\int_{C_{2}[R]} & f\left(x\left(\cdot, t_{1}\right), \cdots, x\left(\cdot, t_{n}\right)\right) d x \\
= & \int_{C_{1}[a, b]} \stackrel{(n)}{\cdots} \int_{C_{1}[a, b]} f\left[\sqrt{\frac{t_{1}-t_{0}}{2}} y_{1}, \sqrt{\frac{t_{1}-t_{0}}{2}} y_{1}+\sqrt{\frac{t_{2}-t_{1}}{2}} y_{2}, \cdots\right. \\
& \left.\sqrt{\frac{t_{1}-t_{0}}{2}} y_{1}+\cdots+\sqrt{\frac{t_{n}-t_{n-1}}{2}} y_{n}\right] d y_{1} \cdots d y_{n}
\end{aligned}
$$

If we let

$$
F_{\sigma}(x) \equiv F\left\{g_{\sigma}\left[x\left(\cdot, t_{1}\right), \cdots, x\left(\cdot, t_{n}\right) ; \cdot, \cdot\right]\right\}
$$

we obtain

$$
\begin{aligned}
& \int_{C_{2}[R]} F_{\sigma}(x) d x \\
& \quad=\int_{C_{1}[a, b]} \stackrel{(n)}{\cdots} \int_{C_{1}[a, b]} F\left\{g_{\sigma}\left[\sqrt{\frac{t_{1}-t_{0}}{2}} y_{1}, \cdots, \sum_{k=1}^{n} \sqrt{\frac{t_{k}-t_{k-1}}{2}} y_{k}\right]\right\} d y_{1} \cdots d y_{n} .
\end{aligned}
$$

It is clear that $\lim _{\text {norm } \sigma \rightarrow 0} F_{\sigma}(x)=F(x)$ for all $x \in C_{2}[R]$ and since $F$ is bounded we may apply Lebesgue's convergence theorem to obtain (6.1).

\section{REFERENCES}

1. J. E. Bearman, Rotations in the product of two Wiener spaces, Proc. Amer. Math. Soc., 3 (1952), 129-137.

2. R. H. Cameron and W. T. Martin, Evaluation of various Wiener integrals by use of certain Sturm-Liouville differential equations, Bull. Amer. Math. Soc., 51 (1945), 73-89.

3. J. E. Kuelbs, Integration on Spaces of Continuous Functions, Thesis, University of Minnesota, 1965.

4. Measures on $C(Y)$ when $Y$ is a compact metric space, Proc. Amer. Math. Soc., 18 (1967), 248-254.

5. J. Yeh, Wiener measure in a space of functions of two variables, Trans. Amer. Math. Soc., 95 (1960), 433-450.

Received March 16, 1973. Research sponsored by N.S.F. Grant GP 28732. 



\section{PACIFIC JOURNAL OF MATHEMATICS}

EDITORS

RICHARD ARENS (Managing Editor)

University of California

Los Angeles, Calıfornia 90024

R. A. Beaumont

University of Washington

Seattle, Washington 98105
J. DugundJI

Department of Mathematics

University of Southern California

Los Angeles, California 90007

D. Gilbarg and J. Milgram

Stanford University

Stanford, California 94305

\section{ASSOCIATE EDITORS}
E. F. BECKENBACH
B. H. NeumanN
F. WOLF
K. YOSHIDA

\section{SUPPORTING INSTITUTIONS}

UNIVERSITY OF BRITISH COLUMBIA

CALIFORNIA INSTITUTE OF TECHNOLOGY

UNIVERSITY OF CALIFORNIA

MONTANA STATE UNIVERSITY

UNIVERSITY OF NEVADA

NEW MEXICO STATE UNIVERSITY

OREGON STATE UNIVERSITY

UNIVERSITY OF OREGON

OSAKA UNIVERSITY
UNIVERSITY OF SOUTHERN CALIFORNIA

STANFORD UNIVERSITY

UNIVERSITY OF TOKYO

UNIVERSITY OF UTAH

WASHINGTON STATE UNIVERSITY

UNIVERSITY OF WASHINGTON

AMERICAN MATHEMATICAL SOCIETY NAVAL WEAPONS CENTER 


\section{Pacific Journal of Mathematics}

\section{Vol. 55, No. $1 \quad$ September, 1974}

Robert Lee Anderson, Continuous spectra of a singular symmetric

differential operator on a Hilbert space of vector-valued functions . . . $\quad 1$

Michael James Cambern, The isometries of $L^{p}(X, K) \ldots \ldots \ldots \ldots \ldots . . \ldots$

R. H. Cameron and David Arne Storvick, Two related integrals over spaces of continuous functions ................................

Gary Theodore Chartrand and Albert David Polimeni, Ramsey theory and

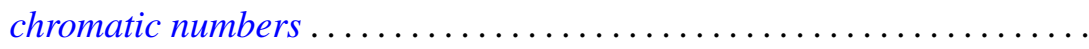

John Deryck De Pree and Harry Scott Klein, Characterization of collectively compact sets of linear operators ...................

John Deryck De Pree and Harry Scott Klein, Semi-groups and collectively compact sets of linear operators ....................... 55

George Epstein and Alfred Horn, Chain based lattices.............. 65

Paul Erdős and Ernst Gabor Straus, On the irrationality of certain series . . 85

Zdeněk Frolík, Measurable uniform spaces................... 93

Stephen Michael Gagola, Jr., Characters fully ramified over a normal

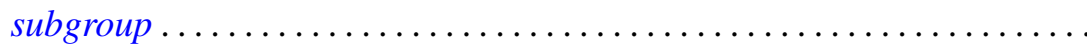

Frank Larkin Gilfeather, Operator valued roots of abelian analytic

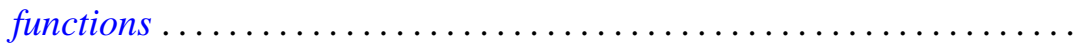

D. S. Goel, A. S. B. Holland, Cyril Nasim and B. N. Sahney, Best approximation by a saturation class of polynomial operators

James Secord Howland, Puiseux series for resonances at an embedded

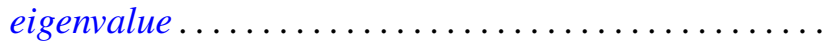

David Jacobson, Linear GCD equations .................

P. H. Karvellas, A note on compact semirings which are multiplicative

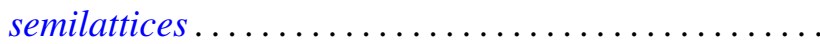

Allan Morton Krall, Stieltjes differential-boundary operators. II . .

D. G. Larman, On the inner aperture and intersections of convex sets

S. N. Mukhopadhyay, On the regularity of the $P^{n}$-integral and its application to summable trigonometric series ....... .

Dwight Webster Read, On $(J, M, m)$-extensions of Boolean algebras ....

David Francis Rearick, Multiplicativity-preserving arithmetic power series.

Indranand Sinha, Characteristic ideals in group algebras

Charles Thomas Tucker, II, Homomorphisms of Riesz spaces . . .

Kunio Yamagata, The exchange property and direct sums of indecomposable injective modules. 